\title{
Nonlinear Surface Plasmon Polaritons at the Interfaces Between Isotropic Media and Anisotropic Left-Handed Materials
}

\author{
G. T. Adamashvili* \\ Max-Planck-Institut fur Physik Komplexer Systeme, Nothnitzer Str. 38, D-01187 Dresden, Germany
}

\begin{abstract}
Linear and nonlinear surface plasmon polaritons propagating along the interfaces between isotropic media and uniaxially anisotropic left-handed materials are investigated. A transition layer sandwiched between the connected media is described using a model of a two-dimensional gas of semiconductor quantum dots. The conditions of the existence of surface TM-modes when the components of the permittivity and permeability tensors of the anisotropic left-handed materials are simultaneously negative are determined. Explicit analytical expressions for a surface optical twodimensional soliton of self-induced transparency in the presence of single and biexciton transitions are given. Numerical simulations for surface solitons are given for different layered systems. It is shown that the total energy flow of the surface solitons depends on the parameters of the quantum dots and the connected media.
\end{abstract}

Keywords: Surface plasmon polaritons, left-handed materials, solitons, quantum dots.

\section{INTRODUCTION}

Surface electromagnetic waves, which are also known as surface plasmon polaritons (SPPs), can propagate along the boundary surface of different media, in a region of frequencies where the permittivities of the two connected media have opposite signs [1,2]. Characteristic peculiarities of these waves are strong enhancement and spatial confinement of the electromagnetic field of the wave near the interface, while they decay exponentially in the directions perpendicular to the wave vector. The properties of surface waves have attracted much interest in the context of nanooptics and diverse applications [3,4]. In left-handed materials (LHMs), the interest in SPPs was connected in the beginning with the strong impact of SPPs on the image resolution of an LHM flat lens [5]. Later, SPPs in LHM have been considered for a wider class of wave phenomena [6-8]. Very recently, they have been used for a experimental realization of nonmagnetic cloaking in the visible frequency range, since the SPP field has only one polarization state [9].

Different properties of surface waves in isotropic LHMs have been studied $[2,4]$. But the LHMs that have been used in different experiments are often anisotropic, and the analysis of the symmetry of the LHMs have shown that they have uniaxial anisotropy. The properties of SPPs propagating in uniaxial anisotropic LHMs are significantly different than in isotropic LHMs. In the general case of uniaxial anisotropic media, unlike for isotropic LHMs, the directions of energy flow cannot be in the exactly opposite direction of the wave vector [10].

Two kinds of uniaxial anisotropic LHMs can be considered. The first, so called indefinite media, are the uniaxial

\footnotetext{
*Address correspondence to this author at the Max-Planck-Institut fur Physik Komplexer Systeme, Nothnitzer Str. 38, D-01187 Dresden, Germany; Tel/Fax: +99532222072; E-mail: gadama@parliament.ge
}

Permanent address: Technical University of Georgia, Kostava str., 77, Tbilisi, 0179, Georgia anisotropic LHMs which are characterized by permittivity and permeability tensors whose principal components do not have the same sign $[11,12]$. The second type of uniaxial anisotropic LHMs are metamaterials where all principal components of the permittivity and permeability tensors are negative $[13,14]$. The optical properties of these two uniaxial anisotropic LHMs are significantly different.

The conditions of the existence of linear surface waves at the interfaces between different isotropic media and indefinite media have been studied [12]. In layered systems, where one of the connected media is an indefinite material, nonlinear SPPs also have been considered [15]. But the properties of the SPPs propagating at the interfaces between different isotropic media and uniaxial anisotropic LHMs where all principal components of the permittivity and permeability tensors are negative have not been investigated.

Embedding semiconductor quantum dots (SQDs) in these layered structures makes them very promising systems for investigations of optical resonance phenomena in plasmonics [16]. SQDs are ideal realizations of zero-dimensional quantum confined systems and have led to the observation of a large number of novel and interesting physical effects which are promising for different applications [17]. The attractivity of SPPs lies in the fact that they can effectively confine the optical excitation in a nanoscale region near the interface and thus mediate strong optical interactions within this region. The resonance interaction between SPPs and SQDs can lead to different resonance linear and nonlinear optical phenomena for SPPs, such as, resonance absorption (Beer's law), Rabi oscillations, self-induced transparency (SIT), electromagnetically induced transparency, and SQDbased laser [18].

Under the condition of SIT, which in the beginning has been considered for atomic systems, resonance nonlinear waves can be formed when a nonlinear coherent interaction takes place via Rabi oscillations of the carrier density, whose conditions 
$\omega \mathrm{T} \gg>1, T<<T_{1,2}$,

are fulfilled. In attenuator media, the steady-state $2 \pi$ pulse (soliton) and in an amplifier medium, the steady-state $\pi$ pulse are generated [19]. Here, $\mathrm{T}$ and $\omega$ are the width and the carrier frequency of the pulse, $\mathrm{T}_{1}$ and $\mathrm{T}_{2}$ are the longitudinal and transverse relaxation times of the SQDs.

SIT for SQDs has been considered for plane waves [20,21] and waveguide modes [22] as well as for SPPs in conventional media [23], in isotropic LHMs [24,25], and in indefinite LHMs [15]. The propagation of SPPs in uniaxial anisotropic LHMs with simultaneously negative principal components of the permittivity and permeability tensors is absolutely different from the earlier investigated cases.

Using the approach advanced in the previous work for indefinite media [15], the purpose of the present work is to theoretically investigate the condition of the existence and the processes of formation of SPPs and surface solitons propagating along the interface between different kinds of isotropic media and uniaxial anisotropic LHMs with simultaneously negative principal components of the permittivity and permeability tensors. For the investigation of solitons of SPPs, we consider the resonance transition monolayer of a two-dimensional gas of inhomogeneously broadened SQDs sandwiched on the interface. The special limiting cases for linear SPPs will be considered. Numerical simulations for SPPs are given for solitons of SPPs in layered structures in the cases when we consider different isotropic media/uniaxial anisotropic LHMs with a transition monolayer of SQDs. We consider four cases of isotropic media which are of physical interest: gallium arsenide, metallic media, single-negative materials (SNMs), and double-negative materials (DNMs). A SNM (magnetic or $\mu$ negative material) is a material where only magnetic permeability has a negative real value, and a DNM is a metamaterial with simultaneously negative real parts of the electric permittivity and magnetic permeability $[12,26]$.

\section{BASIC EQUATION}

We consider the propagation of surface TM-modes along the interface between the isotropic media and the uniaxial anisotropic LHM with simultaneously negative principal components of the electric permittivity and magnetic permeability tensors, for the case when the optical pulse with width $\mathrm{T}$ and frequency $\omega \gg T^{-1}$ is propagating along the positive direction of the $\mathrm{z}$ axis. On the flat border of division $(x=0)$ between the two connected media, a thin transition layer with thickness $d$ containing a small concentration of SQDs having the polarization

$$
\vec{P}(x, y, z)=\vec{e}_{p} p(z, t) \delta(x),
$$

is present, where $\vec{e}_{p}$ is the polarization unit vector along the $\mathrm{z}$ axis. The semi-spaces are divided into $\mathrm{x}<0$ and $\mathrm{x}>0$, with the isotropic medium (medium I) with electric permittivity $\varepsilon_{1}(\omega)$ and magnetic permeability $\mu_{1}(\omega)$ and the LHM (medium II), respectively. For simplicity, we consider only uniaxial anisotropic LHMs with simultaneously negative principal components of the permittivity and permeability tensors.
If we take the optical axis of the LHM $O$ parallel to the interface of the two media along the $\mathrm{z}$ axis and assume that both the electric permittivity $\hat{\varepsilon}_{2}$ and the magnetic permeability $\hat{\mu}_{2}$ tensors are uniaxially anisotropic, we have [27]:

$$
\hat{\varepsilon}_{2}=\left(\begin{array}{ccc}
\varepsilon_{x x} & 0 & 0 \\
0 & \varepsilon_{y y} & 0 \\
0 & 0 & \varepsilon_{z z}
\end{array}\right), \hat{\mu}_{2}=\left(\begin{array}{ccc}
\mu_{x x} & 0 & 0 \\
0 & \mu_{y y} & 0 \\
0 & 0 & \mu_{z z}
\end{array}\right) .
$$

In the considered case, the wave vector $\vec{k}$ is directed along the optical axis of the uniaxial anisotropic LHM $O$ and therefore, the vectors of the electric $\vec{E}$ and magnetic $\vec{H}$ fields and the wave vector constitute a left-handed triplet of vectors [10]. For uniaxial anisotropy, the media are isotropic in the plane perpendicular to the optical axis $O$ and consequently $\varepsilon_{\perp}=\varepsilon_{x x}=\varepsilon_{y y}$ and $\mu_{\perp}=\mu_{x x}=\mu_{y y}$ with the conditions $\varepsilon_{z z} \neq \varepsilon_{\perp}$ and $\mu_{z z} \neq \mu_{\perp}$. The quantities $\varepsilon_{z z}, \mu_{z z}$ and, $\varepsilon_{\perp}, \mu_{\perp}$ are the permittivity and permeability in the directions parallel and perpendicular to the optical axis $O$, respectively. For further considerations, it will be more convenient to use the notations $\varepsilon_{x x}$ and $\varepsilon_{z z}$ for the components of the permittivity tensor.

Strictly speaking, the considered system for surface waves is not a LHM, because for SPPs the vector of the electric field $E$ is not perpendicular to the wave vector and therefore the electric field $E$, the magnetic field $H$, and the wave vector $k$ cannot form a strictly left-handed triplet of mutually orthogonal vectors, even when the wave vector is directed along the optical axis $O$. Nevertheless, considering that the term LHM is widely used in the literature (see, e.g., Refs. $[6,7,10]$ and references therein), we will keep this term in the present work.

In the optical region of the spectrum, $d<<\lambda$, where $d$ is the thickness of a transition layer and $\lambda$ is the length of the surface optical wave. Therefore, SIT can be modelled as a SPP propagating along the interface isotropic media/LHM and the infinitely small thickness transition layer (monolayer) with SQDs [15,23].

For a surface TM-mode, the electric field $\vec{E}\left(E_{x}, 0, E_{z}\right)$ lies in the $x z$ plane perpendicular to the boundary of division between the two connected media, and the magnetic field $\vec{H}\left(0, H_{y}, 0\right)$ is directed along the $y$ axis.

For the investigation of the SPP waves, we will follow an approach which is convenient for the consideration of linear as well as nonlinear surface waves. We will present a set of known equations from the previous work [28], which are necessary for understanding the further considerations. The quantities

$U_{1}(x, z, t)=\int U_{1}(\Omega, Q) e^{k_{1}(\Omega, Q) x+i(Q z-\Omega t)} d \Omega d Q$ for $\quad x<0$,

$U_{2}(x, z, t)=\int U_{2}(\Omega, Q) e^{-k_{2}(\Omega, Q) x+i(Q z-\Omega t)} d \Omega d Q$ for $x>0$,

are Fourier-decomposition of the fields. The quantities 
$\kappa_{1}(\Omega, Q)=\sqrt{Q^{2}-\varepsilon_{1}(\Omega) \mu_{1}(\Omega) \frac{\Omega^{2}}{c^{2}}}$,

$\kappa_{2}(\Omega, Q)=\sqrt{\frac{\varepsilon_{z z}(\Omega)}{\varepsilon_{x x}(\Omega)} Q^{2}-\varepsilon_{z z}(\Omega) \mu_{y y}(\Omega) \frac{\Omega^{2}}{c^{2}}}$

the characterize the transverse structure of the SPP and are determined from the Maxwell equations in connected media. The functions $U_{1,2}$ stand for the components $E_{x}, E_{z}, H_{y}, D_{x}, D_{z}, B_{y}$ in both connected media, where $D_{x}, D_{z}$ and $B_{y}$ are the components of the displacement vector and the magnetic induction vector, respectively. We assume translational invariance in the $y$-direction so that all field quantities do not depend on the coordinate $y$.

Taking into account the surface current caused by the presence of the SQDs, the boundary conditions for surface waves at $x=0$ read $[15,28]$ :

$H_{2, y}-H_{1, y}=\frac{4 \pi}{c} \frac{\partial p}{\partial t}, E_{2, z}=E_{1, z}$.

Using Eqs. (2)-(4), we obtain the nonlinear wave equation for the $z$ component of the strength of the electrical field at $\mathrm{x}=0$ in the following form $[15,28]$ :

$\int f(\Omega, Q) E(\Omega, Q) e^{i(Q z-\Omega t)} d \Omega d Q=4 \pi p(z, t)$

where

$$
\begin{aligned}
& f(\Omega, Q)=\frac{\varepsilon_{z z}}{\kappa_{2}}+\frac{\varepsilon_{1}}{\kappa_{1}}, \\
& E_{1, z}(\Omega, Q)=E_{2, z}(\Omega, Q)=E(\Omega, Q)
\end{aligned}
$$

The equation (5) is valid for any dependence of the polarization of the SQDs $p(z, t)$ on the strength of the electrical field at $x=0$. In order to determine the dependence of the polarization $p(z, t)$ on the strength of the electrical field at $x=0$, we have to consider the structure of the energetic levels of the SQDs and the details of the nonlinear interaction of the surface pulse with the SQDs. The SQDa are described by the Liouville equations similarly to a bulk medium, but unlike the latter the SQDs are affected by the field in the thin film (monolayer) at $\mathrm{x}=0$.

We assume that the pulse is tuned to transitions from the ground state $|1\rangle$ of the SQD to the states $|2\rangle$ and $|3\rangle$, with energies $\varepsilon_{1}=0, \varepsilon_{2}=\hbar \omega_{0}=\varepsilon_{\mathrm{x}}+\delta_{\mathrm{x}} / 2$, and $\varepsilon_{3}=\hbar \Omega_{0}=2$ $\varepsilon_{\mathrm{x}}+\delta_{\mathrm{xx}}$, respectively. The quantities $\varepsilon_{\mathrm{x}}=\left(\varepsilon_{2}+\varepsilon_{2}{ }^{\prime}\right) / 2$ and $\varepsilon_{3}$ are the energies of the single-excitonic and biexcitonic states, respectively. $\delta_{\mathrm{x}}=\mathcal{E}_{2}-\boldsymbol{\varepsilon}_{2}$, and $\delta_{\mathrm{xx}}$ are the energies of the exciton fine structure splitting and biexcitonic binding energy (negative if bound), respectively (Fig. 1); $\hbar$ is Planck's constant. In order that $\delta_{\mathrm{x}} / 2 \ll \hbar \omega_{0}$ and $\delta_{\mathrm{xx}} \ll \hbar$ $\left(\Omega_{0}-\omega_{0}\right)$, the 1 to 2 transition and the 2 to 3 transition are very close to each other and to the pulse frequency $\omega$. To avoid the influence of electron-phonon scattering [29], we assume that the pulse excites the system mostly within the zero-phonon line [30]. The energetic spectrum of the quantum dots can be considered as a quasi-equidistant threelevel system in a cascade configuration $\left(\mu_{13}=0\right)$ under off- resonant excitation $\Omega_{0}-\omega_{0}-\omega \neq 0$ and $\omega_{0}-\omega \neq 0$. We assume that the detunings from the resonance $\Omega_{0}-\omega_{0}-\omega$ and $\omega_{0}-\omega$ lie within the bandwidth of the pulse.

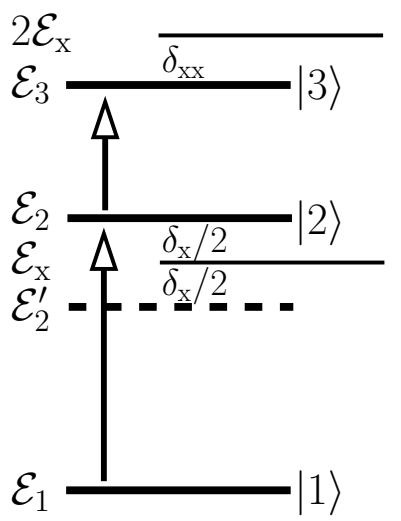

Fig. (1). Schematic of the SQD energetic levels.

The Hamiltonian of the system is given by $[17,21]$ :

$$
H=H_{0}+V \text {, }
$$

where

$$
H_{0}=\hbar \omega_{0}|2><2|+\hbar \Omega_{0}|3><3|,
$$

describes the kinetics of the single-excitonic and biexcitonic states and $V=-\vec{P} \vec{E}$ is the Hamiltonian of the light-quantum dot interaction. Under the assumption of off-resonant excitation with a constant detuning $\Omega_{0}-\omega_{0}-\omega \approx \omega_{0}-\omega=\Delta$, the polarization which is determined by interband transitions occurring in the quantum dots between the three energetic levels is given by

$$
p=n \int g(\Delta)\left(\mu_{12} \rho_{12}+\mu_{23} \rho_{32}\right) d \Delta+c . c .
$$

where $n$ is the uniform quantum dot density, $\mu_{12}=\vec{\mu}_{12} \vec{\varepsilon}, \mu_{23}=\vec{\mu}_{23} \vec{\varepsilon} ; \vec{\mu}_{12}$ and $\vec{\mu}_{23}$ are the dipole elements for the corresponding transitions and $\vec{\varepsilon}$ is the polarization unit vector along $\vec{E}$. We assume the dipole moments to be parallel to each other and to be directed along the $z$ axis, $\mu_{12}=\mu_{23} ; g(\Delta)$ is the inhomogeneous broadening lineshape function resulting from dot size fluctuations. The quantities $\rho_{i j}$ are the matrix elements of the density matrix $\rho$ determined by the Liouville equation

$i \hbar \frac{\partial \rho_{n m}}{\partial t}=\sum_{l}\left(<n|H| l>\rho_{l m}-\rho_{n l}<l|H| m>\right)$,

where $\mathrm{n}, \mathrm{m}, \mathrm{l}=1,2,3$.

\section{SOLUTION OF EQUATIONS}

We can find the solution of the equation (5) following the way presented in the works $[17,21]$. We can simplify Eq. (5) using the method of slowly changing profiles. For this purpose, we represent the functions $E$ and $p$ in the form

$$
E=\sum_{l= \pm 1} \hat{E}_{l} Z_{l}, \quad p=n \mu_{12} \bar{p} Z_{1}+c . c .
$$


where $\hat{E}_{l}$ and $\bar{p}$ are the slowly varying complex amplitudes of the optical electric field and the polarization, respectively, and $Z_{l}=e^{i l(k z-\Omega t)}$. To guarantee that $E$ is a real number, we set $\hat{E}_{l}=\hat{E}_{-l}^{*}=\hat{E}$. This approximation is based on the consideration that the envelope $\hat{E}$ varies sufficiently slowly in space and time as compared to the carrier wave parts, i.e.,

$$
\left|\frac{\partial \hat{E}}{\partial t}\right|<\omega|\hat{E}|,\left|\frac{\partial \hat{E}}{\partial z}\right|<k|\hat{E}| \text {. }
$$

These equations and analogous expressions for $\bar{p}$ are called the slowly varying envelope approximation [19].

It follows from the Liouville equation (8) that the polarization (7) is purely imaginary in the case of perfect resonance $\Delta=0$. But in SQDs, the situation is different and $\Delta$ is not equal to zero. In this case, both the real and imaginary parts of the polarization (7) are not equal to zero.

In these cases, the validity of the simple factorization of the imaginary part of the polarization $\operatorname{Im} p(\Delta)=F(\Delta) \operatorname{Im} p(0)$ is assumed, meaning that the offresonant dipoles respond to the electric field in the same way as the resonant dipoles, but with a detuning-dependent reduction in amplitude

$$
F(\Delta)=\frac{1}{1+(T \Delta)^{2}}
$$

which is called the dipole spectral response function [19].

Substituting the equations (6) and (9) in the wave equation (5), and taking into account the explicit form of the envelope of the polarization (7), which is determined from the Liouville equation, we obtain, after dividing the real and imaginary parts of equation (5), the dispersion law for a propagating surface pulse

$$
f(\omega, k)=0 \text {, }
$$

and a nonlinear wave equation in the form:

$$
\left(\frac{d \hat{E}}{d \zeta}\right)^{2}=T^{-2} \hat{E}^{2}-\frac{\mu_{12}^{2}}{2 \hbar^{2}} \hat{E}^{4},
$$

where the width of the pulse $T$ is determined by the equation

$$
T^{-2}=\frac{4 \pi \mu_{12}^{2} n}{f_{\Omega}\left(\frac{v}{V}-1\right) \hbar} \int g(\Delta) F(\Delta) d \Delta+\mathrm{O}\left(\Delta^{2}\right),
$$

$\mathrm{c}$ is the speed of light in vacuum, $\zeta=t-\frac{z}{V}, V$ is the constant pulse velocity, and

$$
\begin{aligned}
& v=-\frac{f_{Q}}{f_{\Omega}}, \\
& f_{Q}=\left.\frac{\partial f}{\partial Q}\right|_{\Omega=\omega, Q=k}=\left.Q\left(\frac{\varepsilon_{z z}^{2}}{\varepsilon_{x x} \kappa_{2}^{3}}+\frac{\varepsilon_{1}}{\kappa_{1}^{3}}\right)\right|_{\Omega=\omega, Q=k},
\end{aligned}
$$

$$
\begin{aligned}
& f_{\Omega}=\left.\frac{\partial f}{\partial \Omega}\right|_{\Omega=\omega, Q=k}= \\
& \left.\left\{\begin{array}{c}
\frac{d \varepsilon_{1}}{\frac{d \Omega}{\kappa_{1}}}+\frac{\Omega \varepsilon_{1}}{2 c^{2}} \frac{2 \varepsilon_{1} \mu_{1}+\Omega \frac{d\left(\varepsilon_{1} \mu_{1}\right)}{d \Omega}}{\kappa_{1}^{3}}+ \\
\frac{d \varepsilon_{z z}}{\frac{d \Omega}{\kappa_{2}}}-\frac{\varepsilon_{z z}}{2} \frac{\frac{\varepsilon_{z z}}{\varepsilon_{x x}} Q^{2}-\frac{\Omega}{c^{2}}\left[2 \mu_{\perp} \varepsilon_{z z}+\Omega \frac{d\left(\varepsilon_{z z} \mu_{\perp}\right)}{d \Omega}\right.}{\kappa_{2}^{3}}
\end{array}\right\}\right|_{\Omega=\omega, Q=k},
\end{aligned}
$$

where $\omega$ and $k$ are the frequency and wave number of the carrier wave and $v$ is the group velocity of the linear SPP.

The solution of Eq. (12) for the envelope function has the form [19]:

$\hat{E}=\frac{2}{T \mu_{0}} \sec h \frac{t-\frac{z}{V}}{T}$,

where $\mu_{0}=\sqrt{2} \mu_{12} / \hbar$. We can determine the constant velocity of the $2 \pi$ pulse (soliton) of the SPP to be

$$
\frac{v}{V}=\frac{1}{v}+\frac{4 \pi \mu_{12}^{2} n}{f_{\Omega} v \hbar} \int \frac{g(\Delta) d \Delta}{T^{-2}+\Delta^{2}} .
$$

Eqs. (2), (3), (11), (13)-(17) determine the parameters of the surface soliton for any value of $x, z$, and $t$ and show that for the existence of a soliton, it is necessary that the conditions

$$
f_{\Omega}>0, \frac{v}{V}>1
$$

are fulfilled. Parameters of the surface optical solitons depend not only on the SQD parameters and the permittivities of the two interface media, but also on the magnetic permeability $\mu_{\perp}(\omega)$ and its derivative $\left.\frac{d \mu_{\perp}}{d \Omega}\right|_{\Omega=\omega}$ of the LHM, which is unusual in comparison with parameters of the SPP in conventional media [23].

In the absence of a transition layer, the nonlinear polarization $\mathrm{p}=0$, and the right hand side of equation (5) equals zero. In this linear limit, equation (5) has a simple solution with constant amplitude $U_{0}$. The parameters of the linear SPP propagating on the interface between the isotropic media and a LHM for any value of $\mathrm{x}, \mathrm{z}$, and $\mathrm{t}$ and determined from Eqs. (2), (3), (11) and (14) assuming the Fourier-factor $U_{1,2}(\Omega, Q)=U_{0 ; 1,2} \delta(\omega-\Omega) \delta(k-Q)$.

\section{CONDITIONS FOR THE EXISTANCE OF SPPS}

The dispersion relation (11) for SPPs propagating on the interface of the isotropic media and the LHM with a transition layer containing a gas of SQDs is valid for linear as will as nonlinear waves. This statement is valid under the condition that we are neglecting the real part of the polarization $\operatorname{Re} p(\Delta)$. 
Substituting equations (3) in Eq. (11), the dispersion law for linear SPPs and surface solitons of SIT is the same and reads:

$$
k^{2}=\frac{\omega^{2}}{c^{2}} C(\omega)
$$

where

$$
C(\omega)=\varepsilon_{x x} \varepsilon_{1} \frac{\varepsilon_{z z} \mu_{1}-\mu_{\perp} \varepsilon_{1}}{\varepsilon_{z z} \varepsilon_{x x}-\varepsilon_{1}^{2}} .
$$

The quantities $\kappa_{1}$ and $\kappa_{2}$ can be rewritten for further consideration in a more convenient form

$\kappa_{1}^{2}=\frac{\Omega^{2}}{c^{2}} \varepsilon_{1}^{2} D, \kappa_{2}^{2}=\frac{\Omega^{2}}{c^{2}} \varepsilon_{z z}^{2} D$,

where

$D(\omega)=\frac{\varepsilon_{1} \mu_{1}-\mu_{\perp} \varepsilon_{x x}}{\varepsilon_{z z} \varepsilon_{x x}-\varepsilon_{1}^{2}}$.

The SPP can exist only in the region of frequencies $\omega$ for which the quantities $\kappa_{1}, \kappa_{2}$ and $C(\omega)$ are real and positive, i.e., the conditions of the existence of SPPs

$$
C(\omega)>0, \quad D(\omega)>0
$$

are fulfilled.

From Eqs. (19) and (20), we can see that the conditions of the existence of the SPP in the system isotropic medium/ LHM depends not only on the signs of the components of the electric permittivities and the magnetic permeabilities of the connected media, but also on their numerical values.

\section{TOTAL ENERGY FLOW}

The time-averaged Pointing vector of the TM mode $\langle\vec{S}\rangle$, over a period $\frac{2 \pi}{\omega}$, which is associated with the energy flow of the pulse, has $z$ components in isotropic medium 1 and the LHM- medium 2 of the following form:

$$
\begin{gathered}
\left\langle S_{1, z}\right\rangle=\frac{c^{2} k}{8 \pi \omega \varepsilon_{1}}\left|\hat{H}_{1}\right|^{2} e^{2 \kappa_{1} x}, \\
\left\langle S_{2, z}\right\rangle=\frac{c^{2} k}{8 \pi \omega \varepsilon_{x x}}\left|\hat{H}_{2}\right|^{2} e^{-2 \kappa_{2} x} .
\end{gathered}
$$

The corresponding total energy flow is

$N=\int_{-\infty}^{0}\left\langle S_{1, z}\right\rangle d x+\int_{0}^{+\infty}\left\langle S_{2, z}\right\rangle d x=\frac{\omega C}{16 \pi k}\left(\frac{\left|\hat{H}_{1}\right|^{2}}{\varepsilon_{1} \kappa_{1}}+\frac{\left|\hat{H}_{2}\right|^{2}}{\varepsilon_{x x} \kappa_{2}}\right)$.

Equation (21) has a general form and is valid for any boundary condition for the strength of the magnetic field of the SPP. When the transition layer is absent, the boundary condition for the envelopes of the strength of the magnetic field of the surface TM-mode has the form

$\hat{H}_{1}=\hat{H}_{2}$, and Eq. (21) simplifies

$$
N=\frac{\omega C}{16 \pi k}\left(\frac{1}{\varepsilon_{1} \kappa_{1}}+\frac{1}{\varepsilon_{x x} \kappa_{2}}\right)\left|\hat{H}_{1}\right|^{2} .
$$

The total energy flow for a linear SPP is positive or negative, depending on the sign of the quantity $\frac{1}{\varepsilon_{1} \kappa_{1}}+\frac{1}{\varepsilon_{x x} \kappa_{2}}$.

When a resonance transition layer is present, the connection between $\hat{H}_{1}$ and $\hat{H}_{2}$ follows from the boundary condition (4):

$$
\left|\hat{H}_{2}\right|^{2}=\left|\hat{H}_{1}\right|^{2}+i R\left(\bar{p}^{*} \hat{H}_{1}-\bar{p} \hat{H}_{1}^{*}\right)+R^{2}|\bar{p}|^{2},
$$

where

$$
R=\frac{4 \pi \mu_{12} \omega n}{c}
$$

From Eq. (23), it is evident that the resonance transition layer, i.e., the availability of SQDs, has an influence on the total energy flow of the surface TM-mode which depends on the parameter $R$ and the polarization $\bar{p}$.

In the considered case, the quantities $\hat{H}_{1}$ and $\hat{H}_{2}$ are real functions, and hence Eq. (23) simplifies

$$
\hat{H}_{2}^{2}=\hat{H}_{1}^{2}+i R \hat{H}_{1} \operatorname{Im} \bar{p}+R^{2}|\bar{p}|^{2} .
$$

Consequently, when the resonance transition layer is present, the total energy flow depends on the parameters of the SPP, the SQDs, and the connected media.

\section{NUMERICAL ESTIMATIONS}

Precious metallic nanostructures are used in optical LHMs, and therefore the losses are significant for surface optical waves in LHMs. Silver is known to have significantly lower losses than other metals at optical frequencies. The plasma frequency for silver is $\omega_{p}=2 \pi \times 2.18 \cdot 10^{15} \mathrm{~s}^{-1}$ and the damping frequency $\gamma=2 \pi \times 5.08 \cdot 10^{12} s^{-1}$ [31]. On the other hand, a planar array of split-ring resonators can be fabricated on a gallium arsenide (GaAs) substrate [32]. On the surface or at the boundary of the semiconductors (for instance, GaAs or InAs) with another medium, a small concentration of SQDs can be grown. Therefore, for the consideration of the properties of SPPs,it will be expedient to begin in the layered system gallium arsenide/uniaxial anisotropic LHM with simultaneously negative principal components of the permittivity and permeability tensors, which is prepared on the basis of silver and a small concentration of SQDs sandwiched on the interface between the connected media.

In metals and LHMs, SPPs occur when the carrier frequency $\omega$ is below the plasma frequency $\omega_{p}$. For the coherent interaction of the pulse of the surface wave with the medium, the duration of the pulse should be much shorter than the characteristic plasmonic oscillation damping 
time $\frac{2 \pi}{\gamma}$. Solitons of SPPs in regular and indefinite media are investigated when one of the connected media is a semiconductor with SQDs [15]. SQDs are promising objects for the amplification of SPPs [33]. The transverse relaxation times of quantum dots, which are of the order of nanoseconds to several tens of picoseconds [34], are longer than the characteristic plasmonic oscillation damping time $\frac{2 \pi}{\gamma}$. Therefore, pulses of SPPs with durations which are of the order of up to several tens of femtoseconds which are used for investigations of coherent effects in metals can be used to investigate SIT of SPPs. On the other hand, the spectral width of the pulse has to be much smaller than $\omega$,

so that the envelope approximation (10) is appropriate and valid for pulses with width $\mathrm{T} \geq 20$ fs. Consequently, a pulse with a width in the interval $\left(\frac{2 \pi}{\gamma}, 20 \mathrm{fs}\right)$, which is acceptable for investigations of coherent processes for nonresonance nonlinear SPPs in LHMs [6,7], also satisfies Eq. (1), the conditions of SIT in SQDs.

We consider several physically interesting examples of layered systems by means of numerical methods. For the numerical simulation, we use typical parameters for the pulses, materials, and SQDs:

$T=150 \times 10^{-15} s, \omega=\pi \times 10^{15} \mathrm{~Hz}, \mu_{12}=0.8 \times 10^{-17}$ esu cm,

$n=5 \times 10^{10} \mathrm{~cm}^{-2}, \mu_{\perp}=-1, \hbar \delta^{*}=60 \mathrm{meV}$,

where $\hbar \delta^{*}$ is the full-width half-maximum inhomogeneous broadening of the SQDs.
Case (a). First, we consider the layered GaAs/LHM system, in which a small concentration of SQDs is sandwiched on the interface between the connected media. In this system, medium $\mathrm{I}$ is gallium arsenide with the parameters $\varepsilon_{1}=9.9, \mu_{1}=1$.

For medium II, we consider a uniaxial anisotropic LHM with simultaneously negative principal components of the permittivity and permeability tensors. The permittivity component $\varepsilon_{x x}=1-\frac{\omega_{p}^{2}}{\omega^{2}}=-18$, which corresponds to the plasma frequency for silver $\omega_{p}$. For the other nonzero permittivity component $\varepsilon_{z z}$ we consider two different cases:

Case (a.1). $\varepsilon_{z z}=-1$. In this case, after numerical calculations, we obtain

$V=-2.28 \times 10^{10} \mathrm{~cm} / \mathrm{s}, v=-2.29 \times 10^{10} \mathrm{~cm} / \mathrm{s}$,

$f_{\Omega}=2.48 \times 10^{-20} \mathrm{~cm} \cdot \mathrm{s}$,

$C=19.8, D=0.1, k=0.46 \times 10^{6} \mathrm{~cm}^{-1}$,

$k_{1}=0.330 \times 10^{6} \mathrm{~cm}^{-1}, k_{2}=0.333 \times 10^{6} \mathrm{~cm}^{-1}$,

For linear SPPs, the total energy flow $N=-3613\left|\hat{H}_{1}\right|^{2}$.

Because the parameters $\mathrm{C}, \mathrm{D}, \kappa_{1}$ and $\kappa_{2}$ have positive values, the conditions of the existence of linear SPPs, Eq. (20), are fulfilled. For the existence of soliton of SIT for a SPP it will be necessary that the additional conditions Eqs. (1) and (18) are satisfied.

Using these conditions, we can construct a plot of the general form of the $z$ component of the electric field of the

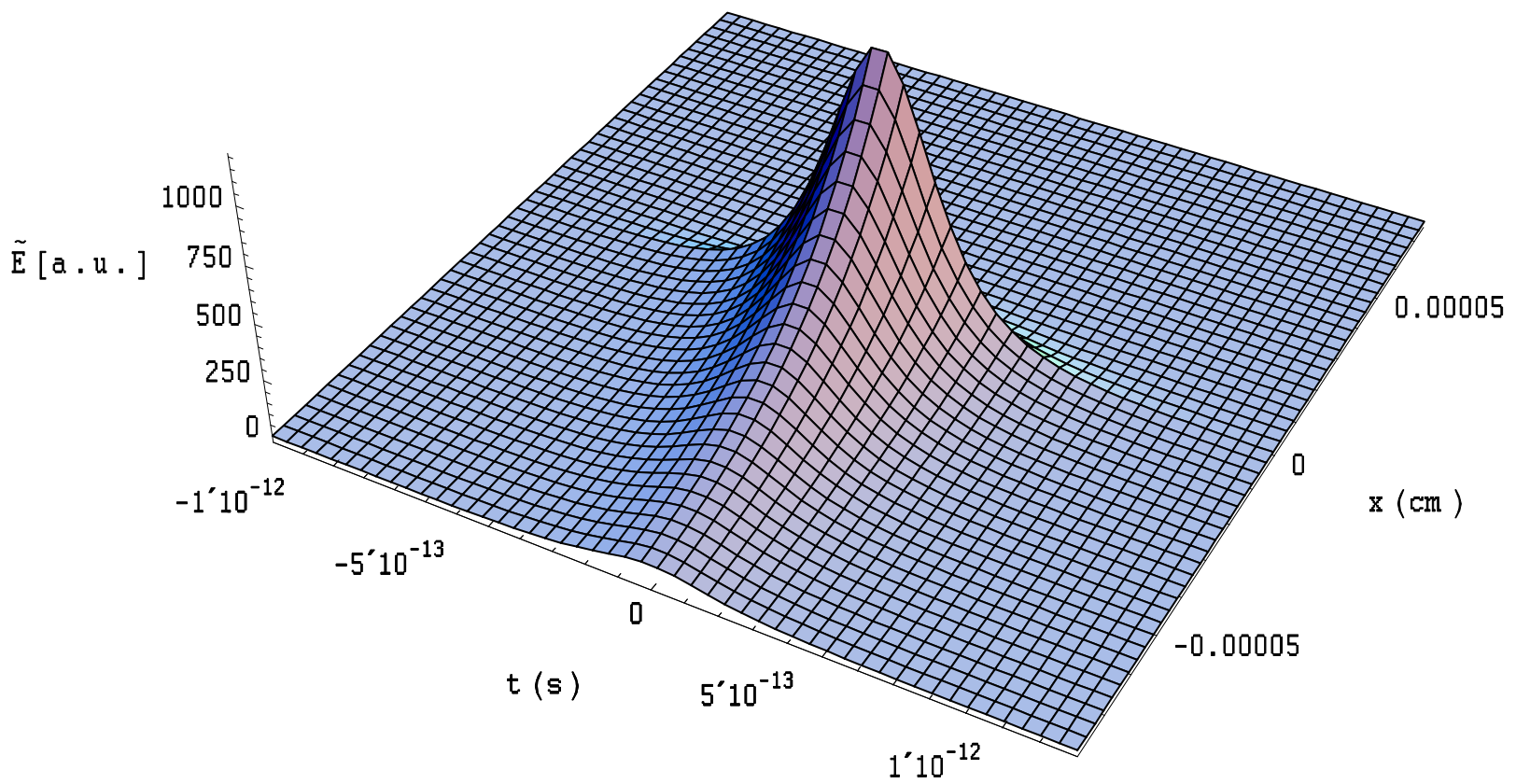

Fig (2). Plot of the $z$ component of the electric field at a fixed value of the $z$ coordinate, showing the envelope of the soliton of a SPP $E$ and the transverse structure of the TM mode in the layered structure gallium arsenide/ LHM with simultaneously negative principal components of the permittivity and permeability tensors and when the resonance transition monolayer containing the ensemble of SQDs is present. Along the $x$-axis, the soliton amplitude decays exponentially as one moves away from the boundaries at $\mathrm{x}=0$. At $\mathrm{x}=0$, the shape of the soliton corresponds to the hyperbolic-secant solution of Eq. (16) (see Fig. 3). The plot corresponds to the Case (a.1) for which $\varepsilon_{z z}=-1$. 


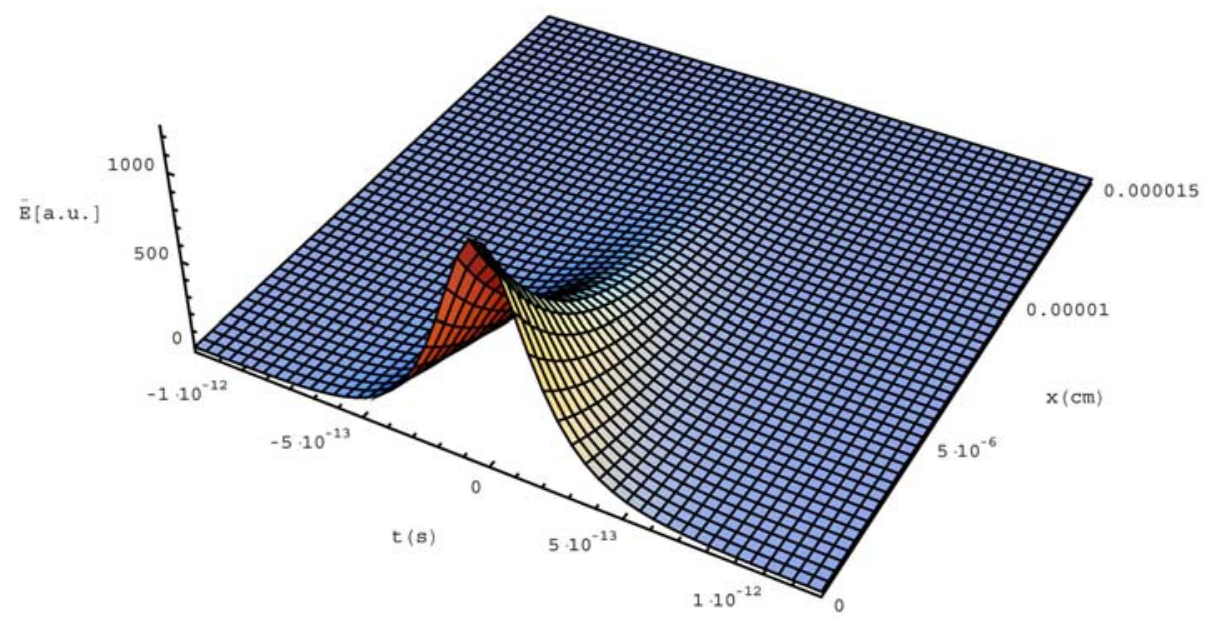

Fig. (3). Plot of the $z$ component of the envelope of the electric field $E$ at a fixed value of the $z$ coordinate (see Fig. 2) with a cut of the envelope of the soliton at $\mathrm{x}=0$ which corresponds to the hyperbolic-secant solution Eq. (16). The plot corresponds to the Case (a.1), for which $\varepsilon_{z z}=-1$.

two-dimensional soliton of a SPP for a fixed value of $z$ (see Figs. 2 and 3). Here, $\tilde{E}=\bar{E} e^{-\kappa_{1} x}$, for $x \geq 0$ and $\tilde{E}=\bar{E} e^{\kappa_{2} x}$, for $x \leq 0$.

The parameters of the SPP are very sensitive to the properties of the connected media. Indeed, if we change only the parameter $\varepsilon_{z z}$ and take instead of $\varepsilon_{z z}=-1$ the value $\varepsilon_{z z}=-5$ with the same values of all other parameters [Case (a.2)], we obtain

$V=-2.28 \times 10^{8} \mathrm{~cm} / \mathrm{s}, v=-2.38 \times 10^{8} \mathrm{~cm} / \mathrm{s}$, $f_{\Omega}=3.5 \times 10^{-21} \mathrm{~cm} \cdot \mathrm{s}$,

$$
\begin{aligned}
& C=109.7, D=1, \quad k=10^{6} \mathrm{~cm}^{-1}, \\
& k_{1}=10^{6} \mathrm{~cm}^{-1}, k_{2}=0.5 \times 10^{6} \mathrm{~cm}^{-1},
\end{aligned}
$$

Now the total energy flow $N=-53\left|\hat{H}_{1}\right|^{2}$ for linear SPPs.

Comparing the two Cases (a.1) and (a.2), we can see that changing only one parameter $\varepsilon_{z z}$ from $\varepsilon_{z z}=-1$ to $\varepsilon_{z z}=-5$, the parameters of the SPP are changed significantly: the group velocity $\mathrm{v}$, the velocity of the nonlinear pulse $\mathrm{V}$, and the total energy flow $\mathrm{N}$ are decreased by two orders of magnitude $\left(10^{2}\right)$. The form of the soliton also changes significantly (Figs. 2 and 4 ), though at the boundary $(\mathrm{x}=0)$, they have the same forms - the hyperbolic-secant form determined by the solution Eq. (16). For the Case (a.1), a cut

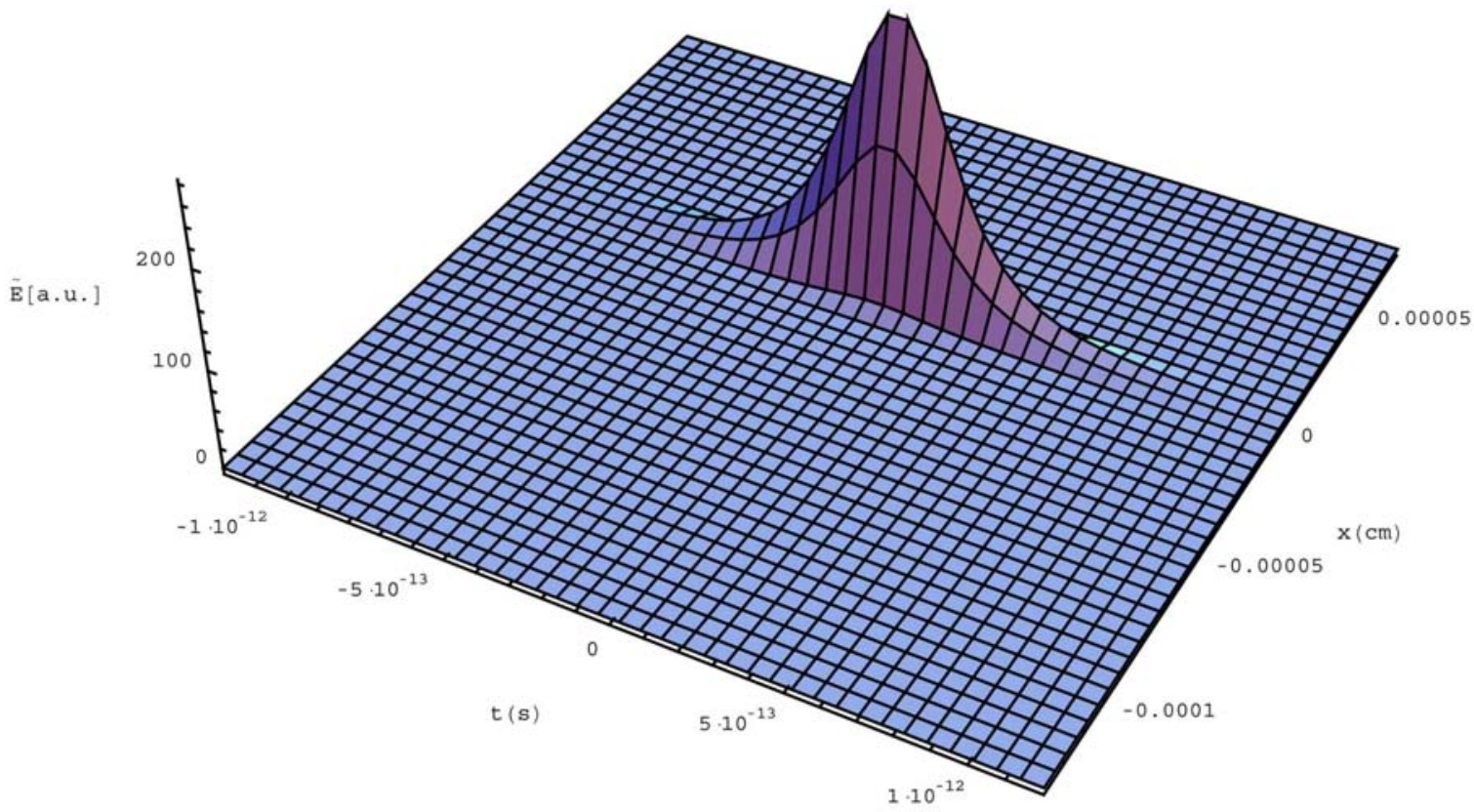

Fig. (4). Plot of the $z$ component of the electric field at a fixed value of the $z$ coordinate showing the envelope of the soliton of a SPP $E$ and the transverse structure of the TM mode in the layered structure gallium arsenide/ LHM with simultaneously negative principal components of the permittivity and permeability tensors and when the resonance transition monolayer containing the ensemble of SQD is present. Along the $\mathrm{x}$-axis, the soliton amplitude decays exponentially as one moves away from the boundary at $\mathrm{x}=0$. The plot corresponds to the Case (a.2), for which $\varepsilon_{z z}=-5$. 


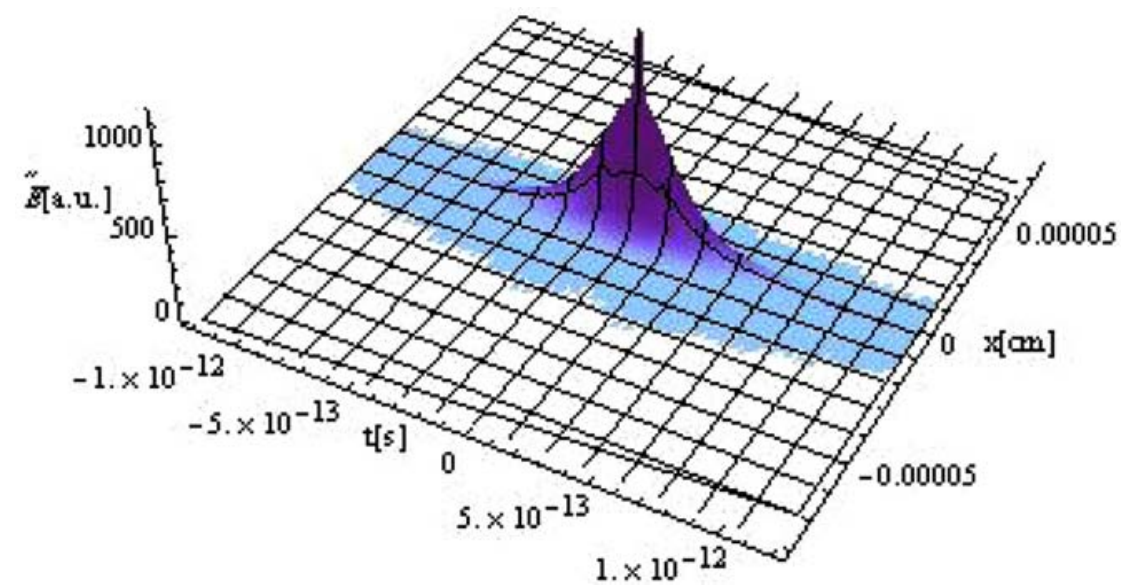

Fig. (5). Plot of the $z$ component of the electric field at a fixed value of the $z$ coordinate showing the envelope of the soliton of a SPP $E$ and the transverse structure of the TM mode in the layered structure a DNM (isotropic LHM) as the isotropic connected medium I / LHM with simultaneously negative principal components of the permittivity and permeability tensors and when the resonance transition monolayer containing the ensemble of SQD is present. Along the $\mathrm{x}$-axis, the soliton amplitude decays exponentially as one moves away from the boundary at $\mathrm{x}=0$. The plot corresponds to the Case (b).

of the envelope of the soliton at $\mathrm{x}=0$ is shown in Fig. (3). For the Case (a.2), the envelope of the soliton at $\mathrm{x}=0$ has the same form.

Case (b). Next, we consider a DNM (isotropic LHM) as the isotropic connected medium I with $\varepsilon_{1}=-10, \mu_{1}=-1$. For the anisotropic LHM, we take $\varepsilon_{z z}=-5$. All other parameters are the same. After calculation, we obtain, (see Fig. 5)

$V=-7.5 \times 10^{9} \mathrm{~cm} / \mathrm{s}, v=-7.9 \times 10^{9} \mathrm{~cm} / \mathrm{s}$,

$f_{\Omega}=3.2 \times 10^{-21} \mathrm{~cm} \cdot \mathrm{s}$,

$C=90, D=0.8, k=996117 \mathrm{~cm}^{-1}$,

$k_{1}=939461 \mathrm{~cm}^{-1}, k_{2}=469731 \mathrm{~cm}^{-1}, \quad N=-1275\left|\hat{H}_{1}\right|^{2}$

Case (c). Next, we consider a SNM (magnetic material) as the isotropic connected medium I with $\varepsilon_{1}=5, \mu_{1}=-1$.
For the anisotropic LHM, we take $\varepsilon_{z z}=-1$. All other parameters are the same. After calculation, we obtain, (see Fig. 6)

$$
\begin{aligned}
& V=-1.1 \times 10^{9} \mathrm{~cm} / \mathrm{s}, \quad v=-1.2 \times 10^{9} \mathrm{~cm} / \mathrm{s}, \\
& f_{\Omega}=1.6 \times 10^{-21} \mathrm{~cm} \cdot \mathrm{s}, \\
& C=77, \quad D=3.2, \quad k=920641 \mathrm{~cm}^{-1}, \\
& k_{1}=949953 \mathrm{~cm}^{-1}, \quad k_{2}=189991 \mathrm{~cm}^{-1}, \quad N=-428\left|\hat{H}_{1}\right|^{2}
\end{aligned}
$$

Case $(d)$. Next we consider a metallic medium as the isotropic connected medium I with $\varepsilon_{1}=-10, \mu_{1}=1$. For the LHM, we take $\varepsilon_{z z}=-5$. All other parameters are the same. After calculation, we obtain, (see Fig. 7)

$V=-3.12 \times 10^{9} \mathrm{~cm} / \mathrm{s}, \quad v=-3.38 \times 10^{9} \mathrm{~cm} / \mathrm{s}$, $f_{\Omega}=1.98 \times 10^{-21} \mathrm{~cm} \cdot \mathrm{s}$

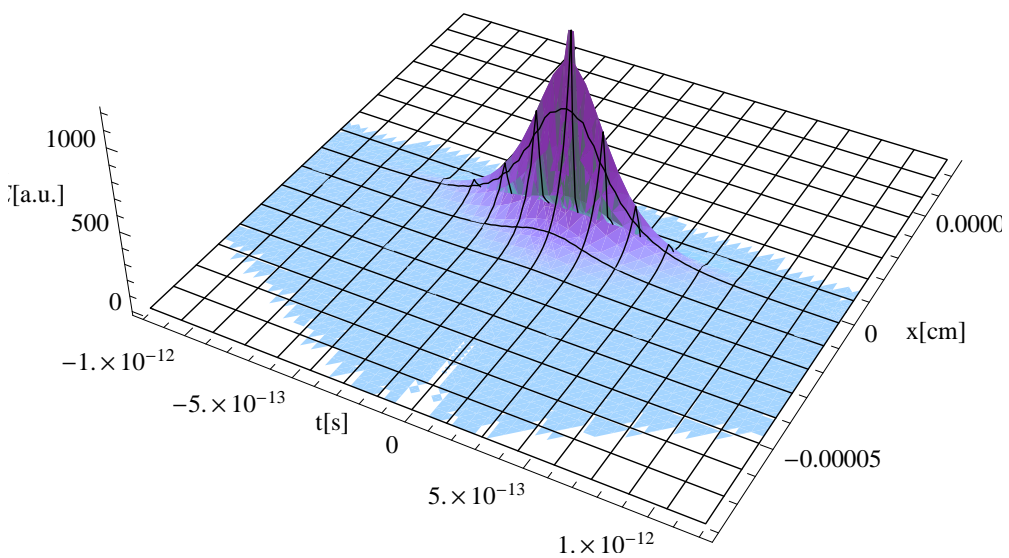

Fig. (6). Plot of the $z$ component of the electric field at a fixed value of the $z$ coordinate showing the envelope of the soliton of a SPP $E$ and the transverse structure of the TM mode in the layered structure a SNM (magnetic material)/ LHM with simultaneously negative principal components of the permittivity and permeability tensors and when the resonance transition monolayer containing the ensemble of SQD is present. Along the $\mathrm{x}$-axis, the soliton amplitude decays exponentially as one moves away from the boundary at $\mathrm{x}=0$. The plot corresponds to the Case (c). 


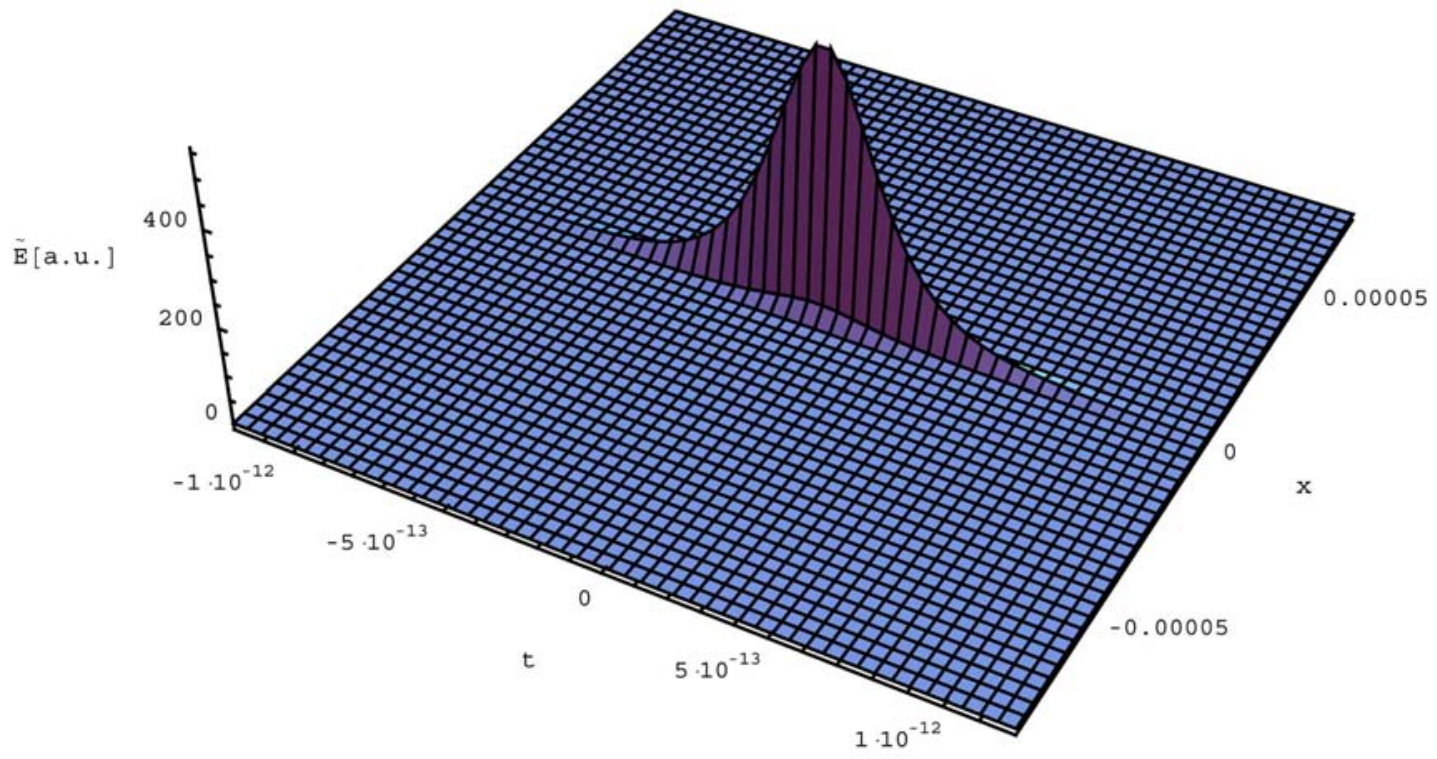

Fig. (7). Plot of the $z$ component of the electric field at a fixed value of the $z$ coordinate showing the envelope of the soliton of a SPP $E$ and the transverse structure of the TM mode in the layered structure a metallic medium / LHM with simultaneously negative principal components of the permittivity and permeability tensors and when the resonance transition monolayer containing the ensemble of SQD is present. Along the $\mathrm{x}$-axis, the soliton amplitude decays exponentially as one moves away from the boundary at $\mathrm{x}=0$. The plot corresponds to the $\operatorname{Case}(d)$, for which $\varepsilon_{z z}=-5$.

$C=271, \quad D=2.8, \quad k=1.7 \times 10^{6} \mathrm{~cm}^{-1}$

$k_{1}=1.76 \times 10^{6} \mathrm{~cm}^{-1}, \quad k_{2}=878409 \mathrm{~cm}^{-1}, \quad N=-1181\left|\hat{H}_{1}\right|^{2}$

These calculations indicate that linear SPPs and solitons of SIT in different layered systems (isotropic media/LHMs) with a SQD transition layer may be experimentally observable.

\section{CONCLUSION}

We have considered the properties of linear and nonlinear SPP TM modes at the interface between an isotropic material and a uniaxial anisotropic LHM with simultaneously negative principal components of the permittivity and permeability tensors. The dispersion relation is determined by equation (19). Conditions for the existence of linear SPPs in these layered structures are determined from equation (20). For the existence of SPP solitons, it is necessary that the conditions Eqs. (1) and (18) are satisfied.

In the absence of a transition layer with SQDs, or when the carrier wave frequency $\omega$ is far away from the frequencies of excitation of the SQDs, the SQDs do not influence the wave processes, and under this condition linear SPPs are formed. The parameters of the linear surface waves for any value of $x, z$ and $t$, are determined from equations (2), (3), (11) and (14). The total energy flow for linear SPPs is positive or negative, depending on the sign of the quantity $\frac{1}{\varepsilon_{1} \kappa_{1}}+\frac{1}{\varepsilon_{x x} \kappa_{2}}$.

When a transition layer with SQDs is sandwiched between an isotropic medium and a LHM, surface resonance solitons of SIT are formed. The explicit shape and the parameters of the surface soliton ( $2 \pi$ pulse) for any value of $x, z$ and $t$ are determined from Eqs. (2), (3), (11)-(17). The parameters of the surface optical solitons depend not only on the permittivity and the magnetic permeability $\mu_{\perp}(\omega)$ and its derivative $\left.\frac{d \mu_{\perp}}{d \Omega}\right|_{\Omega=\omega}$ of the LHM, but also on the SQD parameters. Depending on the parameters of the SPP, the SQDs, and the connected media, the total energy flow for solitons depends on the parameter $R$ and the polarization $\bar{p}$ [Eq. (24)].

The parameters of the surface solitons are very sensitive to a change of the parameters of the connected media. Gallium arsenide, a metallic medium, a SNM, and a DNM are considered as isotropic media. The results of the numerical simulations show that in different isotropic media, the parameters and form of the solitons of a SPP are significantly different [see Eqs. (25)-(30)]. This statement is especially evident when comparing the two Cases (a.1) and (a.2), where we change the value of only one parameter $\varepsilon_{z z}$ : the group velocity, the velocity of the nonlinear pulse, and the total energy flow vary by two orders of magnitude. The forms of the solitons (at $x \neq 0$ ) also change significantly (Figs. 2 and 4 ), though at the boundary $(x=0)$, they have the same forms - the hyperbolic-secant form determined by Eq. (16) (Case (a.1) is shown in Fig. 3).

\section{ACKNOWLEDGEMENT}

None declared.

\section{CONFLICT OF INTEREST}

None declared. 


\section{REFERENCES}

[1] Agranovich VM, Mills DL, Eds. Surface polaritons, electromagnetic waves at surfaces and interfaces. Amsterdam: North-Holland 1982.

[2] Agranovich VM, Gartstein YN. Spatial dispersion and negative refraction of light. Phys Uspekhi 2006; 49: 1029-44.

[3] Raether H. Surface plasmons on smooth and rough surfaces and on gratings. Berlin: Springer 1988.

[4] Zayats AV, Smolyaninov II, Maradudin AA. Nano-optics of surface plasmon polaritons. Phys Rep 2005; 408: 131.

[5] Chen L, He S, Shen L. Finite-size effects of a left-handed material slab on the image quality. Phys Rev Lett 2004; 92: 107404-4.

[6] Agranovich V, Shen Y, Baughman R, Zakhidov A. Linear and nonlinear wave propagation in negative refraction metamaterials. Phys Rev B 2004; 69: 165112-7.

[7] Darmanyan S, Neviere M, Zakhidov A. Nonlinear surface waves at the interfaces of left-handed electromagnetic media. Phys Rev E 2005; 72: 036615-6.

[8] Smolyaninov I, Hung J, Davis C. Imaging and focusing properties of plasmonic metamaterial devices. Phys Rev B 2007; 76: 205424.

[9] Smolyaninov I, Hung J, Davis C. Two-dimensional metamaterial structure exhibiting reduced visibility at 500. Opt Lett 2008; 33: 1342-4.

[10] Hu L, Chui ST. Characteristics of electromagnetic wave propagation in uniaxially anisotropic left-handed materials. Phys Rev B 2002; 66: 085108-7.

[11] Smith D, Kolinko P, Schurig S. Negative refraction in indefinite media. J Opt Soc Am B 2004; 21: 1032-43.

[12] Yan W, Shen L, Ran L, Kong JA. Surface modes at the interfaces between isotropic media and indefinite media. J Opt Soc Am A 2007; 24: 530-5.

[13] Podolskiy VA, Narimanov EE. Strongly anisotropic waveguide as a nonmagnetic left-handed system. Phys Rev B 2005; 71: 201101.

[14] Ilin NV, Kondratiev IG, Smirnov AI. True surface waves guided by metamaterials. Bull Russ Acad Sci Phys 2008; 72: 118.

[15] Adamashvili GT. Surface optical solitons in indefinite media. Phys Lett A 2008; 373: 156-9.

[16] Barnes WL, Dereux A, Ebbesen TW. Surface plasmon subwavelength optics. Nature 2003; 424: 824.

[17] Bimberg D, Grundmann M, Lednetsov L. Quantum Dot Heterostructures. NY: Wiley 1999.

[18] Lin TJ, Chuang WJ, Soofin C, Chen JF. Enhancement of emission from CdSe quantum dots induced by propagating surface plasmon polaritons. Appl Phys Lett 2009; 94: 173506.
[19] Allen L, Eberly JN. Optical resonance and two level atoms. New York-London-Sydney-Toronto: Wiley-Interscience Publisher 1975.

[20] Adamashvili GT, Knorr A. Optical breathers in semiconductor quantum dots. Opt Lett 2006; 31: 74-6.

[21] Adamashvili GT, Weber C, Knorr A, Adamashvili NT. Optical nonlinear waves in semiconductor quantum dots: solitons and breathers. Phys Rev A 2007; 75: 063808-16.

[22] Adamashvili GT, Weber C, Knorr A. Optical solitons in semiconductor quantum dot waveguides. Eur Phys J D 2008; 47 : 113-7.

[23] Adamashvili GT, Knorr A. Surface optical solitons in semiconductor quantum dot layers. Phys Lett A 2007; 367: 220-3.

[24] Adamashvili GT, Adamashvili NT, Peikrishvili MD, Motsonelidze GN, Koplatadze RR. Surface breathers of self-induced transparency in left-handed metamaterials. Tech Phys Lett 2009; 35: 17-21.

[25] Adamashvili GT, Adamashvili NT, Peikrishvili MD, Motsonelidze GN, Koplatadze RR. Surface solitons of self-induced transparency on the interface of arsenide-galium and left-handed materials. Opt Spectrosc 2009; 106: 863-7.

[26] Chettiar UK, Kildishev AV, Hsiao-Kuan Y, et al. Dual-band negative index metamaterial: double negative at $813 \mathrm{~nm}$ and single negative at $772 \mathrm{~nm}$. Opt Lett 2007; 32: 1671-5.

[27] Landau LD, Lifshitz EM. Electrodynamics of continuous media. New York: Pergamon Press 1984.

[28] Maimistov AI, Basharov AM. Nonlinear optical waves. London: Kluwer Academy Publisher 1999.

[29] Krummheuer B, Axt VM, Kuhn T. Theory of pure dephasing and the resulting absorption line shape in semiconductor quantum dots Phys Rev B 2002; 65: 195313-7.

[30] Muljarov EA, Takagahara T, Zimmermann R. Phonon-induced exciton dephasing in quantum dot molecules. Phys Rev Lett 2005; 95: $177405-8$.

[31] Busch K, von Freymann G, Linden S, Mingaleev SF Tkeshelashvili L, Wegener M. Periodic nanostructures for photonics. Phys Rep 2007; 444: 101-202.

[32] Padilla WJ, Taylor AT, Highstrete C, Lee M, Averitt RD. Dynamical electric and magnetic metamaterial response at terahertz frequencies. Phys Rev Lett 2006; 96: 107401-4.

[33] Bergman DJ, Stockman MI. Surface plasmon amplification by stimulated emission of radiation: quantum generation of coheren surface plasmons in nanosystems. Phys Rev Lett 2003; 90: 027402.

[34] Borri P, Langbein W, Schneider S, et al. Ultralong dephasing time in InGaAs quantum dots. Phys Rev Lett 2001; 87: 157401-4

(C) G. T. Adamashvili; Licensee Bentham Open.

This is an open access article licensed under the terms of the Creative Commons Attribution Non-Commercial License (http://creativecommons.org/ licenses/by-nc/3.0/), which permits unrestricted, non-commercial use, distribution and reproduction in any medium, provided the work is properly cited. 